\title{
Evaluating the flow properties of a magnesium ZK60 alloy processed by high-pressure torsion: a comparison of two different miniature testing techniques
}

\author{
Seyed Alireza Torbati-Sarraf ${ }^{\mathrm{a},}$, ${ }^{*}$ Reza Alizadeh ${ }^{\mathrm{b}}$, \\ Reza Mahmudi $^{\mathrm{b}}$, Terence G. Langdon ${ }^{\mathrm{c}}$ \\ ${ }^{a}$ Departments of Aerospace \& Mechanical Engineering and Materials Science, \\ University of Southern California, Los Angeles, CA 90089-1453, U.S.A. \\ ${ }^{b}$ School of Metallurgical and Materials Engineering, College of Engineering, \\ University of Tehran, Tehran, Iran \\ ${ }^{c}$ MaterialsResearch Group, Faculty of Engineering and the Environment, \\ University of Southampton, Southampton SO17 1BJ, U.K.
}

*Corresponding author. E-mail address: torbatis@ usc.edu, Tel: +1 (213) 7404342

\begin{abstract}
A ZK60 magnesium alloy was processed through 5 turns of high-pressure torsion (HPT) at room temperature under an applied pressure of 2.0 GPa to produce a reasonably homogeneous ultrafine microstructure with a grain size of $\sim 700 \mathrm{~nm}$. The potential superplastic behavior of this alloy was investigated by measuring the strain rate sensitivity using two different procedures of miniature tensile testing and miniature shear punch testing (SPT). The tensile experiments were conducted at initial strain rates of $3.0 \times 10^{-5}$ to $1.0 \times 10^{-1} \mathrm{~s}^{-1}$ and the SPT was performed at shear strain rates from $3.3 \times 10^{-3}$ to 3.3 $\times 10^{-1} \mathrm{~s}^{-1}$ at temperatures of 473 and $523 \mathrm{~K}$. It is shown that the strain rate sensitivity index, $m$, has a maximum value of $\sim 0.5$ at intermediate strain rates in both tensile testing and SPT and there was a maximum elongation to failure of $940 \%$ in the tensile testing. The results demonstrate that tensile testing and SPT are both effective procedures in indicating the potential for achieving superplasticity.
\end{abstract}

Keywords: high-pressure torsion, superplasticity, strain rate sensitivity, shear punch testing, ultrafinegrained material 


\section{Introduction}

Superplasticity has many potential applications in various industrial forming operations [1] but the superplastic flow process may be achieved only if two conditions are fulfilled. The first is a high testing temperature, typically of the order of $\sim 0.5 T_{\mathrm{m}}$ where $T_{\mathrm{m}}$ is the absolute melting temperature of the material, and the second is a very small grain size typically smaller than $\sim 10$ $\mu \mathrm{m}[2]$. The poor formability of magnesium alloys at low temperatures is a direct consequence of the limited number of slip systems in their hexagonal close-packed (hcp) structure [3] but nevertheless, under appropriate conditions, superplastic forming may be applied to magnesium alloys to produce complex shapes [4].

Although superplasticity is conventionally examined in uniaxial tensile testing [5], alternative localized and indirect methods are also available such as indentation creep [6], nano indentation [7], impression creep [8] and shear punch testing (SPT) [9], where these various methods may be used to provide indirect information on the advent of superplastic flow. These localized methods are especially beneficial for situations where the amount of material is available only in small quantities as in the samples produced by severe plastic deformation (SPD) processes [10]. In this respect, processing by high-pressure torsion (HPT) is capable of producing exceptional grain refinement [11] but generally the processed samples are in the form of thin disks which are difficult to manipulate in conventional tensile testing. By contrast, these very small samples are ideal for use in alternative indirect procedures such as SPT where only a very small amount of material is needed in order to obtain detailed information on the mechanical properties.

The shear punch technique is based on a blanking operation [12] in which a thin sample, having a thickness in the range of sub-millimeters, is secured between two die halves and a flatended cylindrical punch is driven through the sample to punch out a circular disk. By plotting 
shear stress against normalized displacement, SPT curves are obtained which are generally comparable to those obtained in uniaxial tensile tests. The mechanical properties of the sample, such as the shear yield stress, $\tau_{\mathrm{y}}$, the ultimate shear strength, $\tau_{\mathrm{u}}$, and the elongation values, are then measured directly from the SPT data. It was shown in earlier reports that the data generated by SPT are consistent with, and generally correlate well, with those found using conventional tensile testing for wrought materials $[13,14]$. Nevertheless, there are only very limited reports describing the use of this method for evaluating the mechanical properties of ultrafine-grained (UFG) materials processed by SPD and, without exception, all of these results relate to materials processed by equal-channel angular pressing (ECAP) rather than HPT [15-17].

Recent investigations examined the microstructure and hardness evolution of the ZK60 alloy during HPT processing at room temperature (RT) [7,18-20] and superplastic elongations were reported after HPT within a limited range of strain rates at elevated temperatures [21,22]. However, there has been no attempt to date to measure the essential alloy workability parameters such as the strain rate sensitivity (SRS). Therefore, the objective of the present research was to study the flow properties of an HPT-processed ZK60 alloy using, for comparison purposes, both a miniature tensile testing technique and SPT at elevated temperatures.

\section{Experimental material and procedures}

A ZK60 Mg alloy was used for these experiments where the material was provided as an extruded rod with a chemical composition of $\mathrm{Mg}-5.5 \mathrm{wt} \% \mathrm{Zn}-0.5 \mathrm{wt} \% \mathrm{Zr}$ and an as-received diameter of $10 \mathrm{~mm}$. Samples were sliced into disks perpendicular to the extrusion direction with thicknesses of $\sim 1.2 \mathrm{~mm}$ and these disks were polished and thinned to ultimate thicknesses of $\sim 0.82 \pm 0.02 \mathrm{~mm}$. 
The HPT processing was conducted using an HPT facility with a rotating lower anvil under quasi-constrained conditions [23]. Both the upper and lower anvils contained central spherical depressions with depths of $0.25 \mathrm{~mm}$ and diameters of $10 \mathrm{~mm}$. All processing was conducted at RT using an applied pressure of $2.0 \mathrm{GPa}$ and a constant rotation speed of $1 \mathrm{rpm}$ through total numbers, $N$, of $1 / 2,1$ and 5 turns. Post-processing inspections showed no damage or slippage after processing by HPT through different numbers of revolutions. The average final thicknesses of the disks after processing were $\sim 0.64 \pm 0.03 \mathrm{~mm}$.

Microstructural observations were undertaken near surface areas of the disks located at approximately the mid-radius positions on the cross-sections. Figure 1 shows the location where the processed disks were ion polished by the cross-sectional polishing facility: the directions labeled ND, TD and RD correspond to the normal (torsion axis), tangential and radial directions, respectively, where the shear direction is parallel to TD during HPT processing.

In order to polish the samples for electron back-scatter diffraction (EBSD), a JEOL IB09010CP ion beam cross-sectional polishing machine was used at an operating voltage of 6 $\mathrm{kV}$ for 5 hours. An analytical field emission scanning electron microscope (SEM) JEOL JSM$7001 \mathrm{~F}$ was used at an operating voltage of $7 \mathrm{kV}$ and orientation imaging microscopy (OIM) was utilized to record the experimental data. The grain sizes and the grain size distributions were measured using the OIM software with a count of at least 2000 grains. For the grain size measurements, boundaries having misorientation differences between adjacent measuring points of $2^{\circ}-15^{\circ}$ were set as low-angle grain boundaries (LAGBs) and high-angle grain boundaries (HAGBs) were defined as misorientation differences of $>15^{\circ}$. The pole figures in the EBSD images, measured in the crystallographic orientations of $\langle 0001\rangle,\langle 10 \overline{1} 0\rangle$ and $\langle 2 \overline{1} \overline{1} 0\rangle$, were taken from the semi-oval area of the sample shown in Fig. 1. 
The mechanical properties of the HPT-processed samples were evaluated using the two different testing techniques of conventional tensile testing and SPT methods.

For the tensile experiments, two identical tensile samples were cut from off-center positions of the specimens within the HPT disks with the samples arranged symmetrically to avoid any problems associated with the low strains which are an inherent feature of the central parts of the disks in HPT processing [24]. Electrical discharge machining (EDM) was employed to cut the two miniature tensile specimens from each disk. The gauge lengths of these tensile samples were $1.0 \mathrm{~mm}$ with cross-sectional areas of approximately $1.0 \times 0.64 \mathrm{~mm}^{2}$. An Instron testing machine operating at constant rate of cross-head displacement was used to pull the specimens in tension to failure. Specimens were tested at temperatures of 473 and $523 \mathrm{~K}$ under initial strain rates in the range from $3.0 \times 10^{-5}$ to $1.0 \times 10^{-1} \mathrm{~s}^{-1}$. The recorded load-displacement data were converted to true stress versus true strain and the flow stresses and the elongations to failure, $e_{\mathrm{f}}$, were measured from the stress-strain curves.

For the SPT, the shear punch fixture included a $3.175 \mathrm{~mm}$ diameter flat-ended cylindrical punch and a receiving hole of $3.225 \mathrm{~mm}$ diameter. Tests were conducted at shear strain rates in the range from $3.3 \times 10^{-3}$ to $3.3 \times 10^{-1} \mathrm{~s}^{-1}$ at temperatures of 473 and $523 \mathrm{~K}$ using a screwdriven SANTAM universal testing machine. To achieve thermal equilibrium in the testing setup, the assembly of the specimen and fixture were contained within a split furnace and held for 15 min. The applied load, $P$, was measured automatically as a function of the punch displacement. The data were acquired by a computer to determine the shear stress using the relationship:

$\tau=\frac{P}{\pi D t}$ 
where $t$ is the specimen thickness and $D$ is the average of the punch and die hole diameters. The SPT curves were plotted as the shear stress against the normalized punch displacement of $\delta=h / t$, where $h$ is the displacement. The shear strain rates, $\dot{\gamma}$, were calculated using the equation:

$\dot{\gamma}=\frac{1}{2} \frac{\dot{Z}}{W}$

where $\dot{Z}$ is the punch displacement rate and $W$ is the die-punch clearance. Three separate samples were examined for each condition and the results showed that the variations in the measured strength values were small.

\section{Experimental results}

\subsection{Microstructural and texture features}

Figure 2 provides a comprehensive montage of the grain size distributions and the corresponding EBSD image quality micrographs at the half-radius positions on the disks. Fig. 2a is representative of the alloy in the initial as-extruded condition and inspection reveals fairly equiaxed grains with an almost uniform grain size having a near-normal distribution with an average grain size, $d_{\text {avg }}$, of $\sim 5.0 \pm 2.5 \mu \mathrm{m}$. The microstructures after HPT processing are shown in Fig. 2b-d for 1/2, 1 and 5 turns, respectively, and in these processed conditions there are many highly-refined grains. It is apparent from Fig. $2 b-d$ that a relatively homogenized microstructure develops gradually when increasing the applied torsional straining such that after 5 turns there is a UFG microstructure with reasonable uniformity throughout the disk and an average grain size of $\sim 700 \pm 50 \mathrm{~nm}$. A similar level of grain refinement was reported earlier for a ZK60 alloy processed by ECAP for 6 passes at $473 \mathrm{~K}$ [25]. Similar to the trend observed for the grain sizes, an earlier comprehensive microhardness investigation on the HPT-processed ZK60 alloy 
revealed a gradient of surface hardness values towards a homogeneous distribution with increasing strain [21].

In addition to the grain size and grain size distributions, another important parameter in superplastic materials is the crystallographic texture of the grains. Accordingly, the pole figures of the samples which were obtained from the $\langle 0001\rangle,\langle 10 \overline{1} 0\rangle$ and $\langle 2 \overline{1} \overline{1} 0\rangle$ crystallographic orientations in the TD, RD plane normal to the HPT axis are shown in Fig. 3. These results provide a montage of textures for the unprocessed extruded material prior to HPT followed by samples processed through $1 / 2,1$ and 5 turns. It can be seen that most of the grains typically tend to align their basal $\{0001\}$ planes parallel to the extrusion direction with the $c$-axis parallel to TD and the prismatic $\{10 \overline{1} 0\}$ planes with normals parallel to ND. After processing by $1 / 2$ and 1 turn of HPT at 2.0 GPa there is a gradual evolution towards a fiber texture of basal planes and after 5 turns the majority of grains of different sizes are oriented with their basal $\{0001\}$ planes with the $c$-axis parallel to ND. Recognizing the distinct differences in the critical resolved shear stress $\left(\tau_{\mathrm{CRSS}}\right)$ values of the different slip systems in Mg alloys, it is apparent that the observed differences in the dominant textures of the material shown in Fig. 3 may affect the mechanical behavior when testing in tension and shear deformation. This is examined in the following section after calculation of the corresponding Schmid factor maps for each condition.

Figure 4 shows the Schmid factor maps of the $\{0001\}<11 \overline{2} 0>$ slip system under uniaxial tension and shear deformation for both the extruded (upper row) and HPT-processed condition (lower row) after 5 turns for the ZK60 alloy for tensile testing (on left) and SPT (on right). The corresponding Schmid factor distributions for the same experimental conditions are presented in Fig. 5. It should be noted that, considering the orientation of the samples for the EBSD analysis presented in Fig. 1, the tensile and shear experiments in this study were performed parallel to TD 
and ND, respectively. It can be inferred from the analysis of the Schmid factor data in Figs. 4 and 5 that the average of the Schmid factor is slightly higher in shear deformation by comparison with tensile deformation in both the extrusion and HPT conditions. In addition, it is observed that the average Schmid factor values are close to each other in the extrusion and HPT conditions for similar modes of deformation.

\subsection{A comparison of tensile testing and SPT}

Figure 6 provides a direct comparison between data collected at $473 \mathrm{~K}$ using (a) miniature tensile testing and (b) SPT, where samples were tested in the initial extruded condition and after processing by HPT through 1/2, 1 and 5 turns. The SPT curves in Fig. 6 b consist of a linear elastic part, yielding and then a work hardening region leading to the ultimate shear strength and then a reduction in stress leading to final fracture. These SPT curves are similar in appearance to the tensile curves shown in Fig. 6a. It is apparent from a comparison of the tensile and shear punch results that the strength of the material, in all situations, is lower in SPT. Also, both the SPT and the tensile results demonstrate that the strength of the material decreases with increasing numbers of HPT turns at this elevated testing temperature where this is related to the development of the UFG microstructure combined with modifications in the dominant crystallographic textures of the ZK60 alloy with further torsional straining. This suggests that SPT may provide the capability for evaluating the mechanical properties of materials over a range of grain sizes and textures.

For an evaluation of the possible occurrence of superplasticity, it is necessary to determine the experimental strain rate sensitivity indices since superplastic flow occurs by grain boundary 
sliding and $m \approx 0.5$ [26]. Therefore, the UFG ZK60 alloy processed through 5 turns of HPT was tested under both tensile and shear stresses at $523 \mathrm{~K}$, using initial tensile strain rates in the range from $3.0 \times 10^{-5}$ to $1.0 \times 10^{-1} \mathrm{~s}^{-1}$ and shear strain rates in the range from $3.3 \times 10^{-3}$ to $3.3 \times 10^{-1} \mathrm{~s}^{-}$ 1. The results are shown in Fig. 7a and b for the tensile and shear punch tests, respectively. Both tensile and SPT curves are representative of the generally observed behavior of UFG materials at elevated temperatures, including low strain hardening rates due to microstructural restoration and decreases in the ultimate tensile and shear stresses at lower strain rates. The dependence of the flow stress of the material on the applied rate of deformation in both testing conditions may be used to calculate the strain rate sensitivity and thereby provide an indirect assessment of the possibility of superplastic flow.

The high-temperature flow stress of materials, $\sigma$, is related to the strain rate, $\dot{\varepsilon}$, through a power-law relationship of the form [27]

$$
\frac{\dot{\varepsilon} T}{G}=A\left(\frac{\sigma}{G}\right)^{\frac{1}{m}} \exp \left(\frac{-Q}{R T}\right)
$$

where $A$ is a material parameter, $G$ is the shear modulus, $Q$ is the deformation activation energy, $R$ is the universal gas constant and $T$ is the absolute temperature. Considering the von-Mises yield criterion for pure shear of kinematically hardening materials, this equation can be adapted to evaluate the occurrence of superplastic behavior in SPT by substituting $\dot{\varepsilon}$ by $\dot{\gamma}$ and $\sigma$ by $\tau$ and rewriting as a modified power-law relationship of the form [28]:

$$
\frac{\dot{\gamma} T}{G}=\dot{A}\left(\frac{\tau}{G}\right)^{\frac{1}{m}} \exp \left(\frac{-Q}{R T}\right)
$$

where $A ́$ is a material constant. Thus, since $Q$ is constant at any selected temperature, it follows that $m$ is given by:

$$
m=\left(\frac{\partial \ln \left(\frac{\sigma}{G}\right)}{\partial \ln \left(\frac{\dot{\varepsilon} T}{G}\right)}\right)_{T}
$$


or from the shear relationship by

$$
m=\left(\frac{\partial \ln \left(\frac{\tau}{G}\right)}{\partial \ln \left(\frac{\dot{\gamma} T}{G}\right)}\right)_{T}
$$

The lower section of Fig. 8a shows a plot of the flow stress against the temperaturecompensated strain rate in tensile testing where the strain rate values were normalized to the shear modulus to eliminate the changes in shear modulus with temperature. It is readily apparent that they exhibit a sigmoidal relationship which divides the behavior into three distinct regions, where the observed behavior is identical to that recorded in conventional superplastic materials with slightly larger grain sizes [29]. From the slopes of the line in Fig. 8a, the values of $m$ are estimated as $\sim 0.20, \sim 0.47$ and $\sim 0.14$ in regions I, II and III, respectively. The upper section of Fig. 8a gives the plot of the elongation to failure, $e_{\mathrm{f}}$, plotted against the temperature compensated strain rate where the highest recorded elongation is $940 \%$ which occurs towards the lower end of region II: the sample showing this high elongation is depicted in the inset in Fig. 8a. An elongation of $940 \%$ provides unambiguous evidence for the occurrence of true superplastic flow in this alloy since superplasticity requires elongations of at least $400 \%$ [5] and the results show that, as in conventional superplastic alloys, superplastic flow occurs over about two orders of magnitude at intermediate strain rates. It is noted also that there is no visible necking within the gauge length of the specimen pulled to $940 \%$ and this is also consistent with the requirements for superplastic flow [30].

Similar evidence for superplastic behavior is visible in the SPT results in Fig. 8b where the normalized shear stress is plotted against the temperature compensated shear strain rate. For these conditions there is again a sigmoidal relationship dividing the behavior into three regions with values of $m$ of $\sim 0.26, \sim 0.49$ and $\sim 0.17$ in regions I, II and III, respectively. These values of $m$ are very similar to those obtained using conventional tensile testing in Fig. 8a, and therefore, it 
is concluded that SPT provides a simple and expedient method for determining the strain rate sensitivities in metallic materials and checking on the possibilities of superplastic flow

\section{Discussion}

Many investigations have studied the grain refinement mechanisms of face centered cubic (fcc) materials through SPD processes in which the large grains are subdivided by low-angle boundaries having small misorientations and the subsequent formation of subgrain boundaries leads to high-angle boundaries during further straining of the sample [31-33]. Nevertheless, the grain refinement mechanism for hcp metals, such as $\mathrm{Mg}$, appears to be different from fcc metals. Specifically, in hep metals there is an initial formation of a multi-modal grain distribution with a necklace-like microstructure appearing along the original grain boundaries as a result of dynamic recrystallization (DRX) $[33,34]$. A quantitative analysis of the evolution of the grain size distribution presented in Fig. 2 confirms that there is a gradual refinement through HPT processing at room temperature such that the distribution of grain sizes transfers from a peak value at $\sim 2 \mu \mathrm{m}$ after $1 / 2$ turn to a peak closer to $\sim 1 \mu \mathrm{m}$ after 1 turn and then to two distinct peaks after 5 turns of HPT as shown in Fig. 2d.

These results confirm, therefore, that the ZK60 alloy may be successfully processed by HPT at room temperature for the production of a fairly uniform UFG microstructure. This is an important advantage of HPT processing over ECAP where the billets often crack during the processing operation [35]. Nevertheless, the main disadvantage of HPT is the relatively small thin samples that require special miniature testing facilities for an evaluation of the mechanical properties. The present investigation employed two different miniature testing methods and it is 
apparent that SPT provides a simple alternative method for overcoming the difficulties inherent in conventional tensile testing.

Processing by HPT also affects the crystallographic texture evolution in the ZK60 alloy as shown by the pole figures in Fig. 3. The initial texture of $\{$ hki0 $\}$ fiber means that the $\{10 \overline{1} 0\}$ planes in the extruded condition are oriented perpendicular to the extrusion axis and the $\{0001\}$ planes generally lie parallel to the extrusion axis. Therefore, the hcp crystal $c$-axis is in the RDTD plane of the disks before processing by torsion straining in HPT. This texture evolves during processing into a basal fiber texture since the $c$-axis of many hcp crystals tends to rotate and align with the compressive direction with the basal planes parallel to the RD-TD plane of the HPT disks. During torsional straining, there are transformation states at $1 / 2$ and 1 turn which contain two components including the compressed $\{0001\}<u v t w>$ basal fiber and a second fiber. Eventually, a basal fiber texture of $\{0001\}<$ uvtw $>$ is produced after straining through 5 turns and producing the UFG microstructure. These results are consistent with texture results obtained by XRD during HPT processing of Mg alloys at room temperature [7,36]. It is important to note that these distinct differences in the crystallographic orientations of the grains in the HPT and extruded conditions will affect the mechanical behavior of the alloy in the tensile and shear modes of deformation.

The CRSS of magnesium alloys is sensitive to the deformation temperature and decreases with an increase in temperature [37,38]. This effect is more pronounced for the non-basal slip systems by comparison with basal slip so that non-basal slip is more activated at elevated temperatures. By increasing the numbers of active slip systems at elevated temperatures, it may be anticipated that the formability of magnesium alloys will be improved even though, at all temperatures, the basal slip system of $\{0002\}<11 \overline{2} 0>$ remains dominant in $\mathrm{Mg}$ alloys. 
Extensive slip on this system generally leads to an alignment of the basal planes with the deformation direction. In addition to temperature, the mode of deformation will affect the Schmid factor as observed in Figs. 4 and 5. It is apparent from Fig. 5 that the average Schmid factor is higher in shear than in tensile deformation and this indicates that the basal slip system operates relatively easier during shear punch testing along the ND than in tensile testing along the TD. From the present results, it is evident from a comparison of the ultimate tensile and shear strengths given in Figs. 6 and 7 that the material yields more easily in SPT for all experimental conditions.

A comparison of the values of the Schmid factors also provides important information. From Fig. 5 it follows that, while the average Schmid factor is $\sim 0.31$ for the extruded material in the tensile mode, the average value is $\sim 0.29$ for the same mode after 5 turns. Similarly, average Schmid factor values of $\sim 0.35$ and $\sim 0.36$ were obtained in the shear mode for the extruded and HPT-processed material, respectively. It seems, therefore, that the initial extrusion or HPT texture of the material prior to tensile and shear testing has little or no effect on the Schmid factors. Since there is a rotation by $90^{\circ}$ from a prismatic fiber texture in the extruded material to a basal fiber texture in the HPT-processed material, and also the tensile and shear deformation occurs in parallel with TD and ND, respectively, the similarity between the values of the Schmid factors for the extruded and HPT-processed conditions are readily understood. Thus, in the extruded material most of the basal planes are aligned perpendicular to the tensile direction which is TD, whereas in the HPT-processed material the basal planes are aligned perpendicular to ND which is the shear direction. This means that the Schmid factor values should be similar under both conditions. 
The UFG microstructure of the ZK60 alloy gives a high strain rate sensitivity of $m \approx 0.47$ by tensile testing at $523 \mathrm{~K}$ and a maximum elongation to failure of $940 \%$. This confirms the occurrence of superplastic flow and a similar value of $m \approx 0.49$ was obtained by SPT at the same testing temperature. Since these two values of $m$ are almost identical, it follows that the use of SPT provides an excellent procedure for identifying possible ranges of temperature and strain rate wherein superplastic elongations may be attained. Although the SPT method was used earlier for studying superplasticity in different alloy systems processed using SPD techniques $[15-17,39,40]$, the technique was not validated by reference to any alternative and proven measurement technique. In the present experiments, the use of a single alloy processed by HPT for both miniature tensile testing and SPT provides a direct comparison between these diverse testing procedures. Furthermore, the similarities in the measured strain rate sensitivities between these two methods confirms that SPT is a viable procedure for evaluating superplastic behavior even when the material is in the form of the very thin disks that are available after HPT processing.

\section{Summary and conclusions}

1. An extruded ZK60 magnesium alloy was successfully processed through HPT at room temperature to give, after 5 turns of HPT at room temperature, an ultrafine-grained microstructure with an average grain size of $\sim 700 \mathrm{~nm}$.

2. Samples processed by HPT were examined using either conventional miniature tensile testing or shear punch testing (SPT). Both methods gave similar values for the strain rate sensitivity of $m \approx 0.50$ and with a maximum elongation recorded in tensile testing of $940 \%$. This value of $m$ and the high elongation confirms the occurrence of superplasticity. 
3. The ability to record similar and consistent values of the strain rate sensitivity using SPT demonstrates that this procedure, which is easy to perform, provides a simple method for expeditiously evaluating the possible occurrence of superplastic flow in the very small samples available after HPT processing.

\section{Acknowledgements}

This work was supported in part by the National Science Foundation of the United States under Grant No.DMR-1160966 and in part by the European Research Council under ERC Grant Agreement No. 267464-SPDMETALS. 


\section{Reference}

1. A.J. Barnes, Superplastic forming 40 years and still growing, J. Mater. Eng. Perform 16 (2007) 440-454.

2. T.G. Langdon, The mechanical properties of superplastic materials, Metall. Trans. A 13A (1982) 689-701.

3. B.L. Mordike, T. Ebert, Magnesium: properties-applications-potential, Mater. Sci. Eng. A 302 (2001) 37-45.

4. J.A. del Valle, F. Peñalba, O.A. Ruano, Optimization of the microstructure for improving superplastic forming in magnesium alloys, Mater. Sci. Eng. A 467 (2007) 165-171.

5. T.G. Langdon, Seventy-five years of superplasticity: historic developments and new opportunities, J. Mater. Sci. 44 (2009) 5998-6010.

6. R.Mahmudi, H. Mhjoubi, P. Mehraram, Superplastic indentation creep of fine-grained Sn-1\% Bi alloy, Int. J. Modern Phys. B 22 (2008) 2823-2832.

7. I.C. Choi, D.H. Lee, B. Ahn, K. Durst, M. Kawasaki, T.G. Langdon, J.I. Jang, Enhancement of strain-rate sensitivity and shear yield strength of a magnesium alloy processed by high-pressure torsion, Scripta Mater. 94 (2015) 44-47.

8. R.Mahmudi, R. Alizadeh, A.R. Geranmayeh, Enhanced superplasticity in equal-channel angularly pressed Sn-5Sb alloy, Scripta Mater. 64 (2011) 521-524.

9. R. Mahmudi, R. Alizadeh, Sh. Azhari, Strain rate sensitivity of equal-channel angularly pressed Sn-5Sb alloy determined by shear punch test, Mater. Lett. 97 (2013) 44-46.

10. T.G. Langdon, Twenty-five years of ultrafine-grained materials: achieving exceptional properties through grain refinement, Acta Mater. 61 (2013) 7035-7059.

11. A.P. Zhilyaev, T.G. Langdon, Using high-pressure torsion for metal processing: fundamentals and applications, Prog. Mater. Sci. 53 (2008) 893-979.

12. T.M. Chang, H.W. Swift, Shearing of metal bars, J. Inst. Metals 78 (1950) 119-146.

13. R.K. Guduru, K.A. Darling, R. Kishore, R.O. Scattergood, C.C. Koch, K.L. Murty, Evaluation of mechanical properties using shear-punch testing, Mater. Sci. Eng. A 395 (2005) 307-314.

14. R.K. Guduru, A.V. Nagasekhar, R.O. Scattergood, C.C. Koch, K.L. Murty, Finite element analysis of a shear punch test, Metall. Mater. Trans. 37A (2006) 1477-1483.

15. S.A. Torbati-Sarraf, R. Mahmudi, Microstructure and mechanical properties of extruded and ECAPed AZ31 Mg alloy, grain refined with Al-Ti-C master alloy, Mater. Sci. Eng. A 627 (2010) 3515-5320. 
16. S.A. Torbati-Sarraf, R. Mahmudi, Ultra fine-grained AZ31 magnesium alloy obtained by a combination of grain refinement and equal channel angular pressing, Int. J. Mod. Phys. Conf. Ser. 05 (2012) 307-315.

17. R. Alizadeh, R. Mahmudi, A.H.W. Ngan, Y. Huang, T.G. Langdon, Microstructure, texture and superplasticity of a fine-grained $\mathrm{Mg}-\mathrm{Gd}-\mathrm{Zr}$ alloy processed by equal channel angular pressing, Metall. Mater. Trans. 47A (2016) 6056-6069.

18. S.A. Torbati-Sarraf, T.G. Langdon, Mechanical properties of ZK60 magnesium alloy processed by high-pressure torsion, Adv. Mater. Res. 922 (2014) 767-772.

19. H.J. Lee, S.K. Lee, K.H. Jung, G.A. Lee, B. Ahn, M. Kawasaki, T.G. Langdon, Evolution in hardness and texture of a ZK60A magnesium alloy processed by high-pressure torsion, Mater. Sci. Eng. A 630 (2015) 90-98.

20. S.A. Torbati-Sarraf, S. Sabbaghianrad, R.B. Figueiredo, T.G. Langdon, Orientation imaging microscopy and microhardness in a ZK60 magnesium alloy processed by highpressure torsion, J. Alloy Cmpds. 712 (2017) 185-193.

21. S.A. Torbati-Sarraf, T.G. Langdon, Properties of a ZK60 magnesium alloy processed by high-pressure torsion, J. Alloy Compds. 613 (2014) 357-363.

22. S.A. Torbati-Sarraf, S. Sabbaghianrad, T.G. Langdon, Microstructural properties, thermal stability and superplasticity of a ZK60 Mg alloy processed by high-pressure torsion, Letters Mater. 5 (2015) 287-293.

23. R.B. Figueiredo, P.H.R. Pereira, M.T.P. Aguilar, P.R. Cetlin, T.G. Langdon, Using finite element modeling to examine the temperature distribution in quasi-constrained highpressure torsion, Acta Mater. 60 (2012) 3190-3198.

24. A. Loucif, R.B. Figueiredo, M. Kawasaki, T. Baudin, F. Brisset, R. Chemam, T.G. Langdon, Effect of aging on microstructural developments in an $\mathrm{Al}-\mathrm{Mg}-\mathrm{Si}$ alloy processed by high-pressure torsion, J. Mater. Sci. 47 (2012) 7815-7820.

25. R.B. Figueiredo, T.G. Langdon, The development of superplastic ductilities and microstructural homogeneity in a magnesium ZK60 alloy processed by ECAP, Mater. Sci. Eng. A 430 (2006) 151-156.

26. T.G. Langdon, A unified approach to grain boundary sliding in creep and superplasticity, Acta Metall. Mater. 42 (1994) 2437-2443.

27. T.G. Langdon, Creep at low stresses: An evaluation of diffusion creep and Harper-Dorn creep as viable creep mechanisms, Metall. Mater. Trans. 33A (2002) 249-259.

28. R. Alizadeh, R. Mahmudi, T.G. Langdon, Superplasticity of a fine-grained Mg-9Gd-4Y$0.4 \mathrm{Zr}$ alloy evaluated using shear punch testing, J. Mater. Res. Technol. 3 (2014) 228232.

29. H. Ishikawa, F.A. Mohamed, T.G. Langdon, The influenece of strain rate on ductility in the superplastic Zn-22\% Al eutectoid, Phil. Mag. 32 (1975) 1269-1271. 
30. T.G. Langdon, Fracture processes in superplastic flow, Metal Sci. 16 (1982) 175-183.

31. T.G. Langdon, The principles of grain refinement in equal-channel angular pressing, Mater. Sci. Eng. A. 462 (2007) 3-11.

32. S. Sabbaghianrad, M. Kawasaki, T.G. Langdon, Microstructural evolution and the mechanical properties of an aluminum alloy processed by high-pressure torsion, J. Mater. Sci. 47 (2012) 7789-7795.

33. R.B. Figueiredo, T.G Langdon, The nature of grain refinement in equal-channel angular processing: a comparison of representative fcc and hep metals, Int. J. Mater. Res. 100 (2009) 1636-1648.

34. R.B. Figueiredo, T.G. Langdon, Grain refinement and mechanical behaviour of a magnesium alloy processed by ECAP, J. Mater. Sci. 45 (2010) 4827-4836.

35. R.B. Figueiredo, P.R. Cetlin, T.G. Langdon, The processing of difficult-to-work alloys by ECAP with an emphasis on magnesium alloys, Acta Mater. 55 (2007) 4769-4779.

36. Y. Huang, R.B. Figueiredo, T. Baudin, A.L. Helbert, F. Brisset, TG. Langdon, Microstructure and texture evolution in a magnesium alloy during processing by highpressure torsion, Mater. Res. 16 (2013) 577-585.

37. R.E. Reed-Hill, W.D. Robertson, Deformation of Mg single crystals by non-basal slip, Trans. Metall. Soc. AIME 209 (1957) 496-502.

38. E.W. Kelly, W.F. Hosford, Deformation characteristics of textured magnesium, Trans. Metall. Soc. AIME 242 (1968) 654-661.

39. R. Alizadeh, R. Mahmudi, Evaluating high-temperature mechanical behavior of cast $\mathrm{Mg}-$ 4Zn-xSb magnesium alloys by shear punch testing, Mater. Sci. Eng. A 527 (2010) 39753983.

40. R. Alizadeh, R. Mahmudi, P.H.R. Pereira, Y. Huang, T.G. Langdon, Microstructural evolution and superplasticity in an $\mathrm{Mg}-\mathrm{Gd}-\mathrm{Y}-\mathrm{Zr}$ alloy after processing by different SPD techniques, Mater. Sci. Eng. A 682 (2017) 577-585. 


\section{Figure captions}

Fig. 1. Near surface position of the disks for EBSD sample preparation with ion beam crosssectional polishing facility: ND, TD and RD correspond to the normal direction (torsion axis), tangential direction and radial direction, respectively, and the shear direction is parallel to TD.

Fig. 2. Grain size distributions and corresponding EBSD image quality maps of the material taken from the RD-TD planes of disks (a) in the extruded condition and (b) after 1/2 turn, (c) 1 turn and (d) 5 turns of HPT at the mid-radius of the disks.

Fig. 3. The pole figures of the $\{0001\},\{10 \overline{1} 0\}$ and $\{2 \overline{1} \overline{1} 0\}$ crystallographic orientations on the TD-RD plane and normal to the ND of HPT direction from the mid-radius of the extruded disk and HPT-processed disks after 1/2, 1 and 5 turns at $298 \mathrm{~K}$.

Fig. 4. Schmid factor maps of the $\{0001\}<11 \overline{2} 0>$ slip system in (a,b) the extruded condition and $(\mathrm{c}, \mathrm{d})$ after HPT processing for 5 turns under $(\mathrm{a}, \mathrm{c})$ tensile and $(\mathrm{b}, \mathrm{d})$ shear modes of deformation.

Fig. 5. The distributions of the Schmid factors of the $\{0001\}<11 \overline{2} 0>$ slip system in (a,b) the extruded condition and (c,d) after HPT-processing for 5 turns under $(\mathrm{a}, \mathrm{c})$ tensile and $(\mathrm{b}, \mathrm{d})$ shear modes of deformation.

Fig. 6. Plots of (a) true stress versus true strain after pulling in tension to failure at an initial strain rate of $1.0 \times 10^{-3} \mathrm{~s}^{-1}$ and (b) shear stress versus normalized displacement at a shear strain rate of $1.6 \times 10^{-2} \mathrm{~s}^{-1}$ : results obtained at $473 \mathrm{~K}$ for the extruded and HPT conditions.

Fig.7. Plots of (a) true stress versus true strain after pulling in tension to failure and (b) shear stress versus normalized displacement obtained at $473 \mathrm{~K}$ under different initial strain rates for the material processed by 5 turns of HPT.

Fig. 8. (a) Elongation to failure (upper) and normalized flow stress (lower) as a function of temperature-compensated initial strain rate and (b) normalized shear flow stress as a function of temperature-compensated shear strain rate for the alloy processed by HPT through 5 turns and tested at $523 \mathrm{~K}$. 


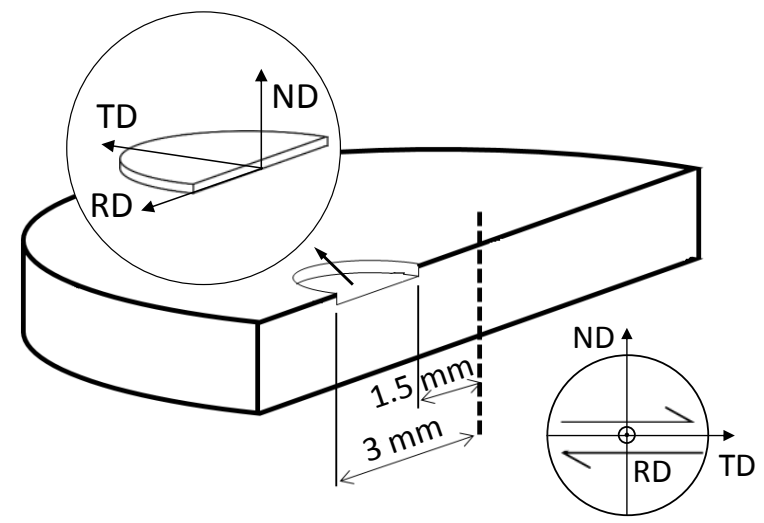

Fig. 1. Near surface position of the disks for EBSD sample preparation with ion beam crosssectional polishing facility: ND, TD and RD correspond to the normal direction (torsion axis), tangential direction and radial direction, respectively, and the shear direction is parallel to TD. 
ZK60

Extruded

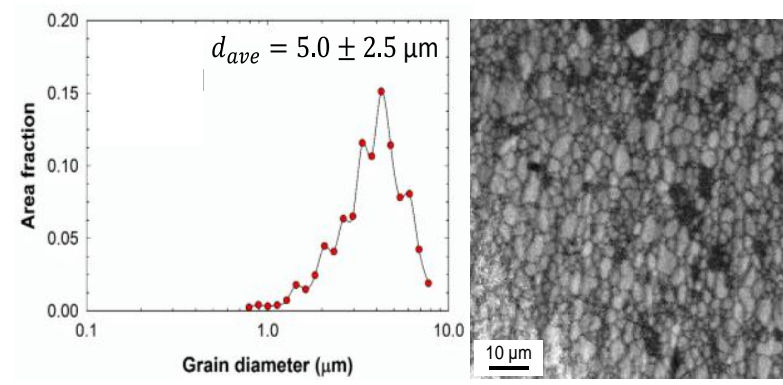

(a)

\section{ZK60}

HPT: 1 turn

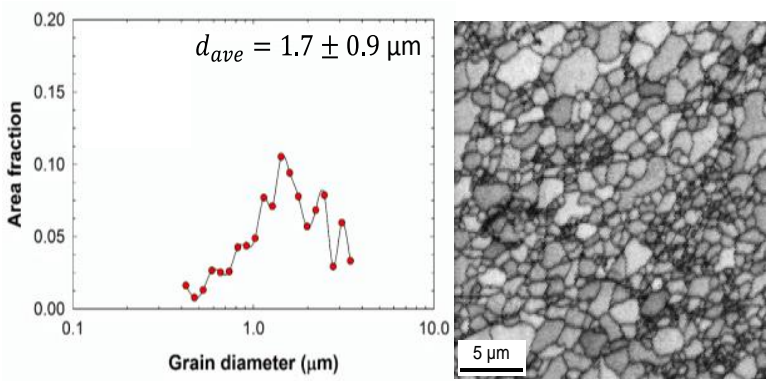

(c)
ZK60

HPT: 1/2 turn

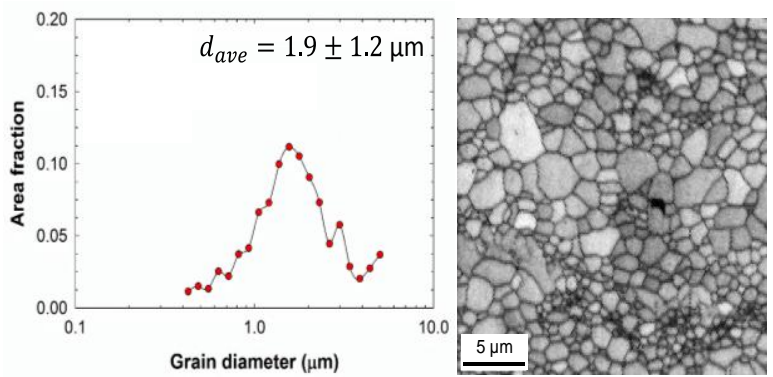

(b)

\section{ZK60}

HPT: 5 turns

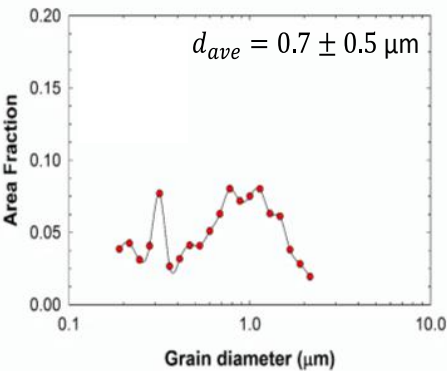

(d)

Fig. 2. Grain size distributions and corresponding EBSD image quality maps of the material taken from the RD-TD planes of disks (a) in the extruded condition and (b) after 1/2 turn, (c) 1 turn and (d) 5 turns of HPT at the mid-radius of the disks. 


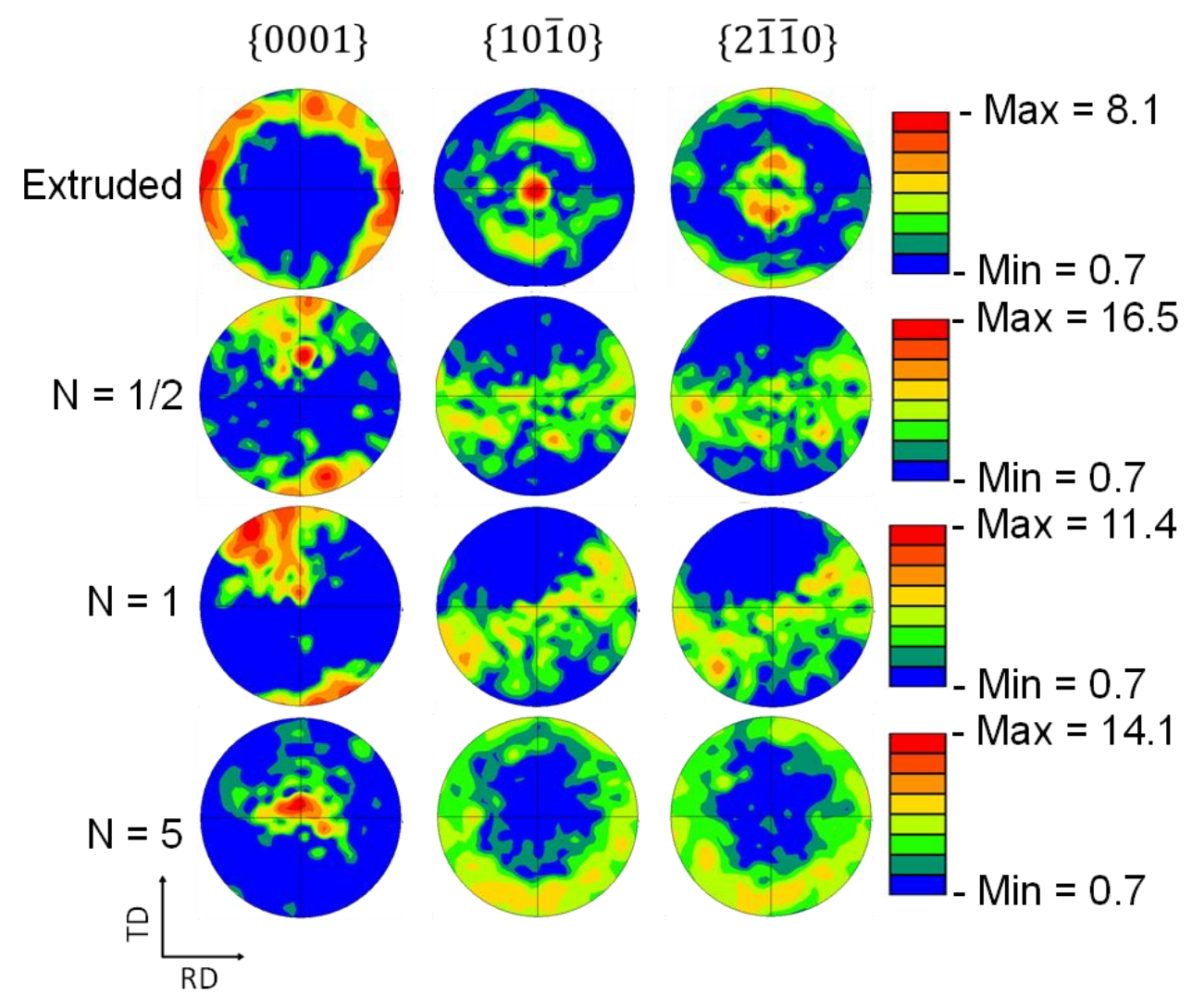

Fig. 3. The pole figures of the $\{0001\},\{10 \overline{1} 0\}$ and $\{2 \overline{1} \overline{1} 0\}$ crystallographic orientations on the TD-RD plane and normal to the ND of HPT direction from the mid-radius of the extruded disk and HPT-processed disks after 1/2, 1 and 5 turns at $298 \mathrm{~K}$. 


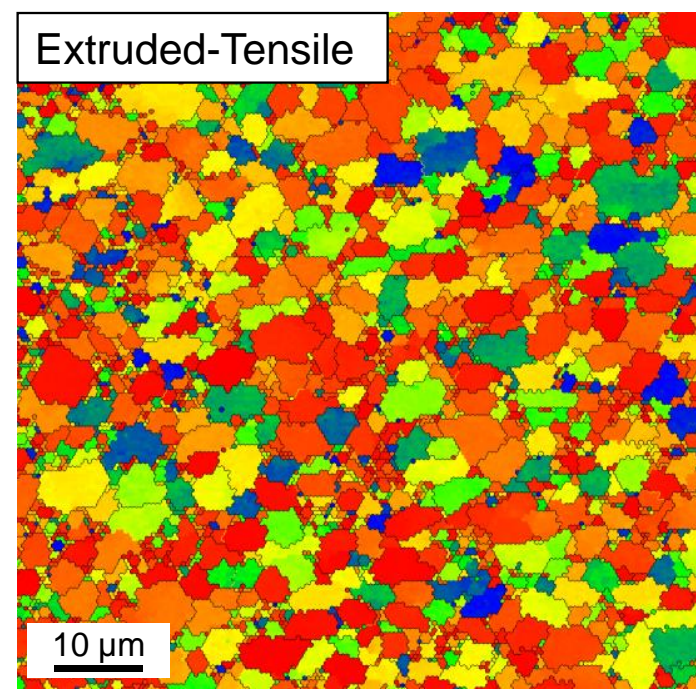

(a)

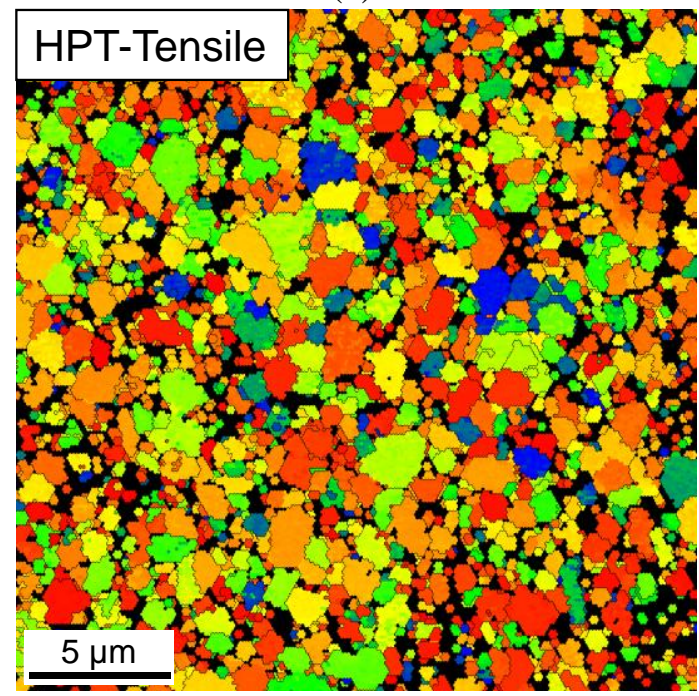

(c)

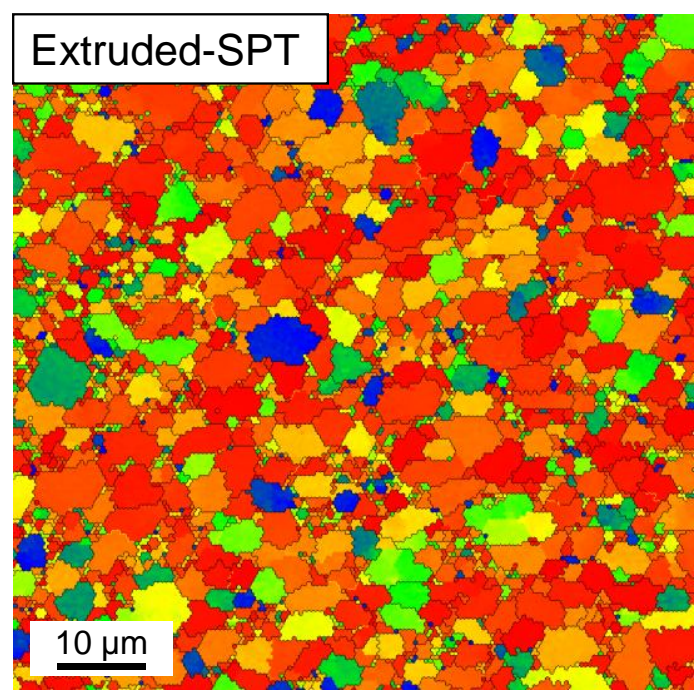

(b)

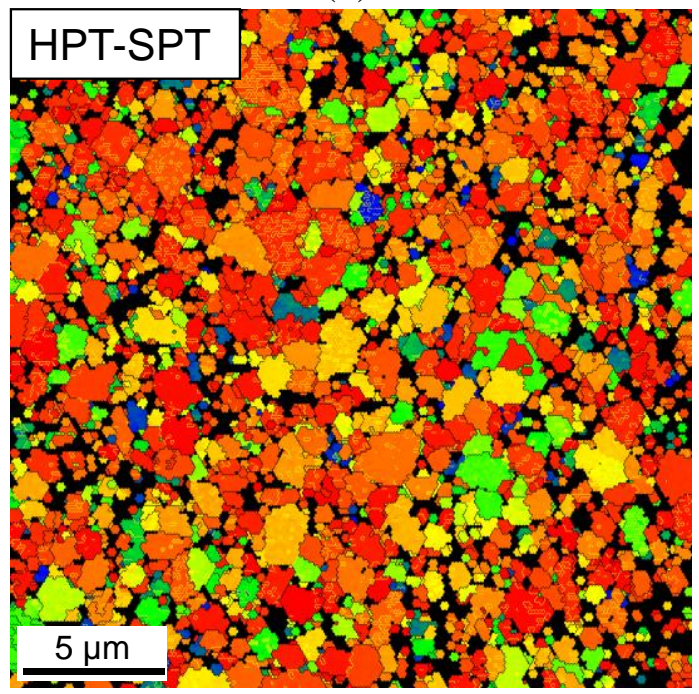

(d)

Fig. 4. Schmid factor maps of the $\{0001\}<11 \overline{2} 0>$ slip system in $(a, b)$ the extruded condition and (c,d) after HPT processing for 5 turns under $(\mathrm{a}, \mathrm{c})$ tensile and (b,d) shear modes of deformation. 


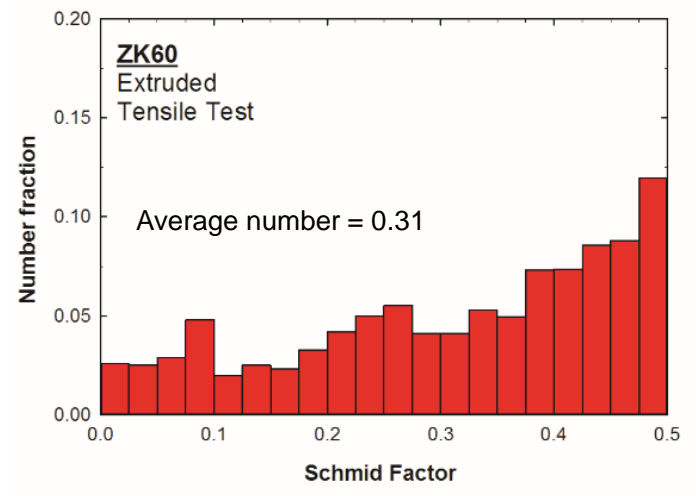

(a)

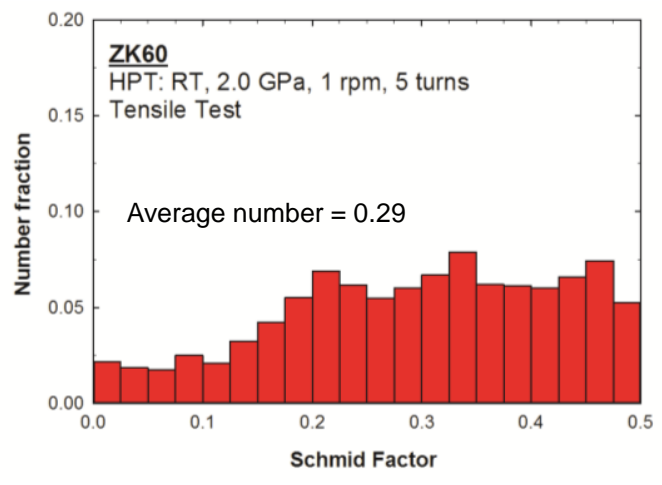

(c)

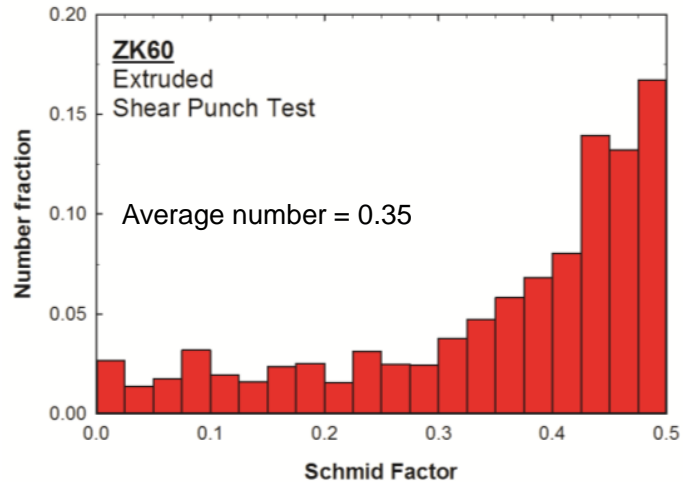

(b)

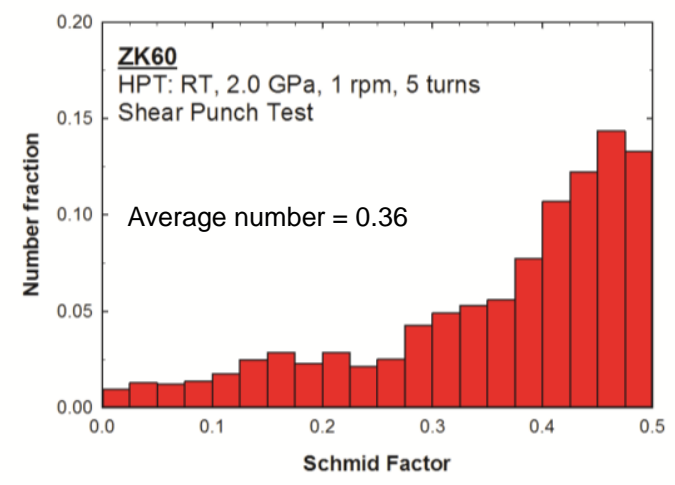

(d)

Fig. 5. The distributions of the Schmid factors of the $\{0001\}<11 \overline{2} 0>$ slip system in $(\mathrm{a}, \mathrm{b})$ the extruded condition and (c,d) after HPT-processing for 5 turns under $(\mathrm{a}, \mathrm{c})$ tensile and $(\mathrm{b}, \mathrm{d})$ shear modes of deformation. 


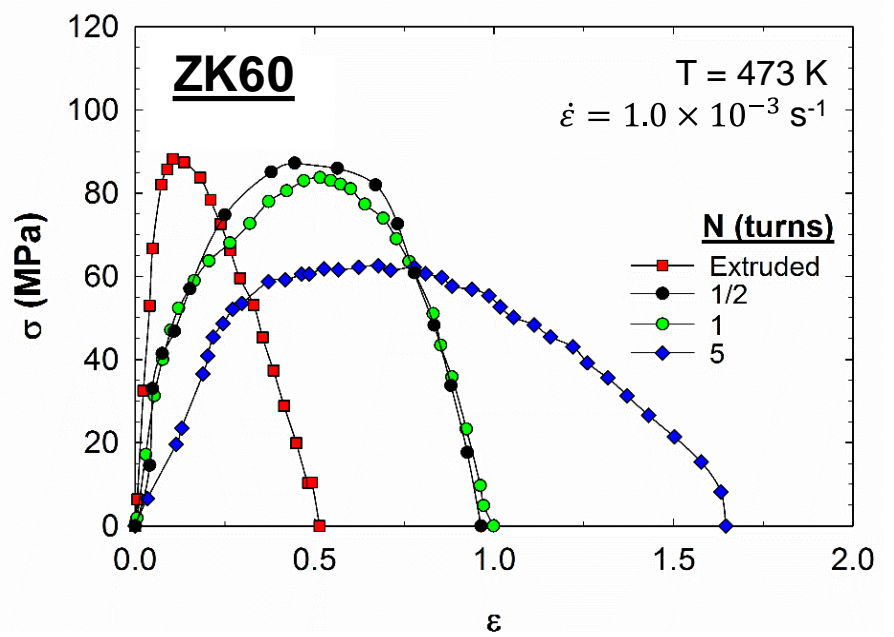

(a)

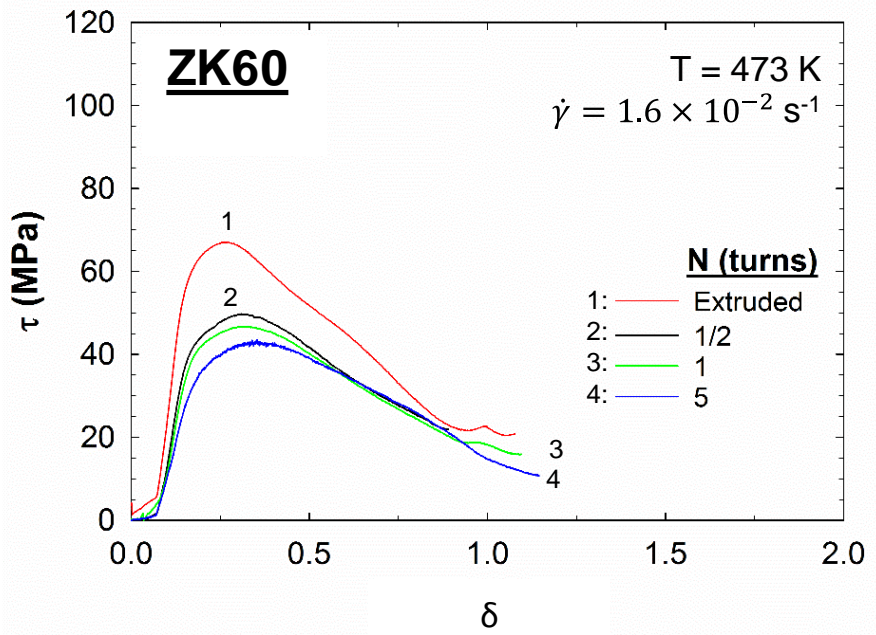

(b)

Fig. 6. Plots of (a) true stress versus true strain after pulling in tension to failure at an initial strain rate of $1.0 \times 10^{-3} \mathrm{~s}^{-1}$ and (b) shear stress versus normalized displacement at a shear strain rate of $1.6 \times 10^{-2} \mathrm{~s}^{-1}$ : results obtained at $473 \mathrm{~K}$ for the extruded and HPT conditions. 


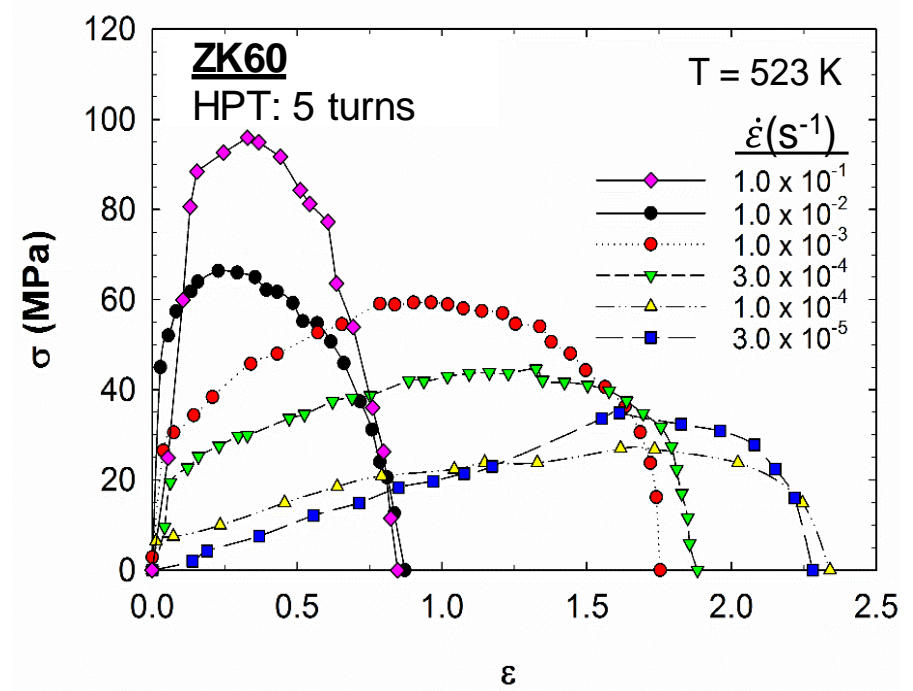

(a)

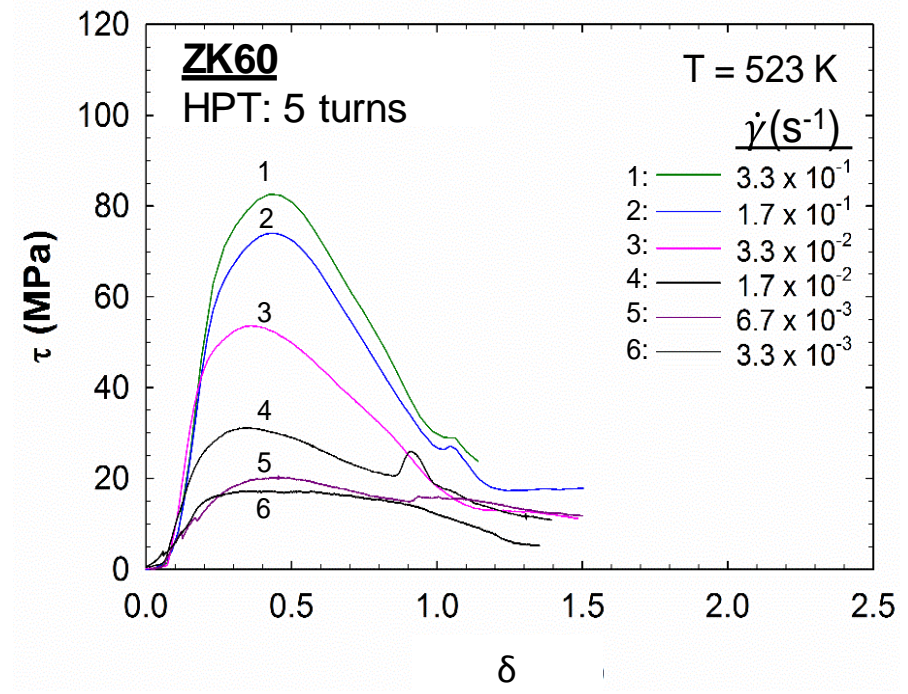

(b)

Fig. 7. Plots of (a) true stress versus true strain after pulling in tension to failure and (b) shear stress versus normalized displacement obtained at $473 \mathrm{~K}$ under different initial strain rates for the material processed by 5 turns of HPT. 


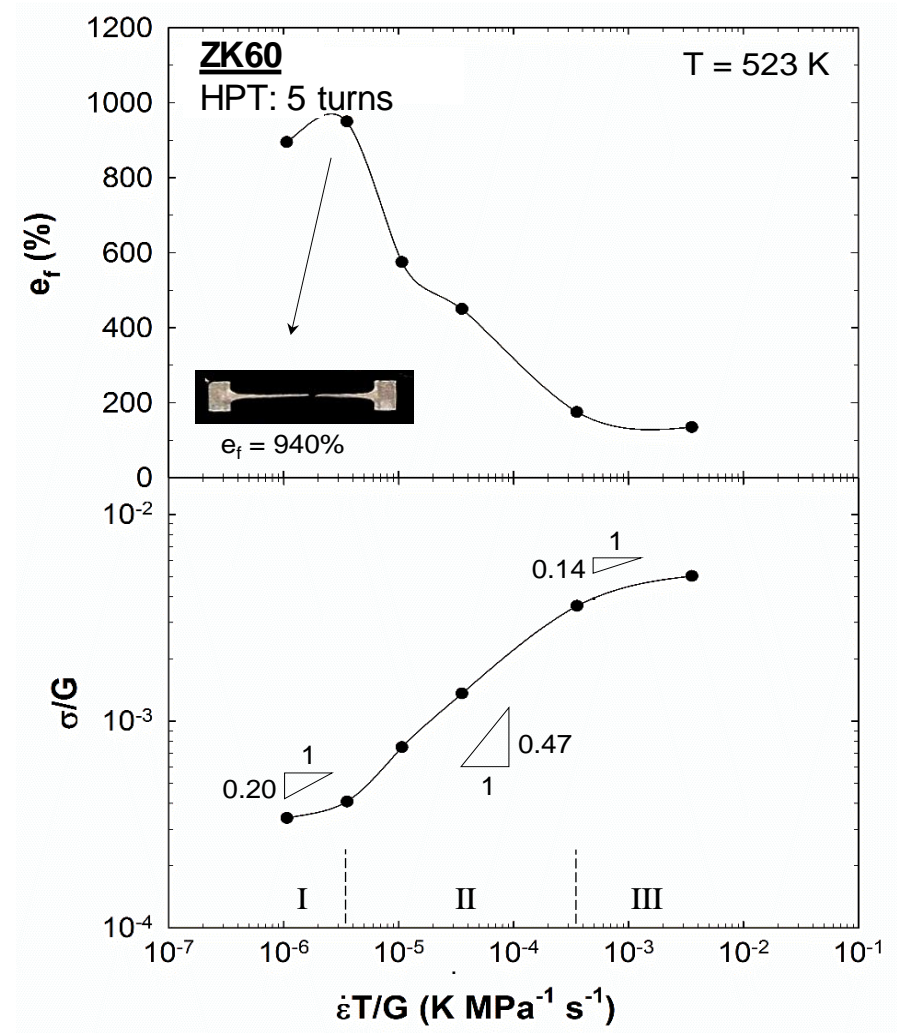

(a)

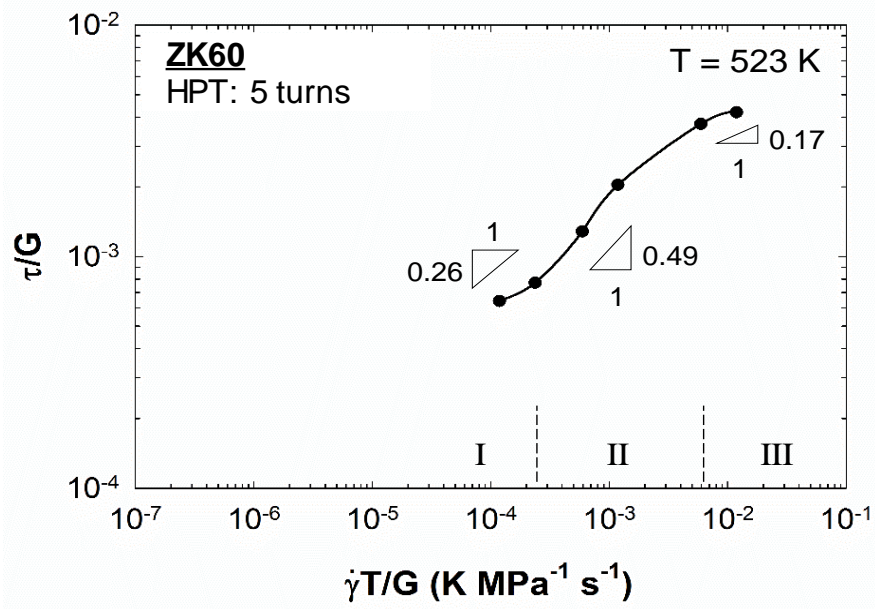

(b)

Fig. 8. (a) Elongation to failure (upper) and normalized flow stress (lower) as a function of temperaturecompensated initial strain rate and (b) normalized shear flow stress as a function of temperature-compensated shear strain rate for the alloy processed by HPT through 5 turns and tested at $523 \mathrm{~K}$. 